\title{
SÊNTIMUS: um Jogo Digital Musical para Crianças com Hipersensibilidade Sonora e Características dos Transtornos do Neurodesenvolvimento
}

\author{
Débora Line Gomes ${ }^{1}$, Cybelle Loureiro ${ }^{1}$, Raquel Prates ${ }^{2}$ \\ ${ }^{1}$ Escola de Música - Universidade Federal de Minas Gerais (UFMG) \\ 31270-010 - Belo Horizonte - MG - Brasil \\ ${ }^{2}$ Departamento de Ciência da Computação - Universidade Federal de Minas Gerais (UFMG) \\ deboralg@ufmg.br, cybelle@musica.ufmg.br, rprates@dcc.ufmg.br
}

\begin{abstract}
This paper describes the research involved in the design and evaluation the game Sêntimus: A Musical Digital Game for Children with Hypersensitivity to Sounds and Features of Neurodevelopmental Disorders. The game was developed based on treatment principles identified in the literature for both diseases, and was evaluated with 5 music therapists and also with 3 children with Neurodevelopmental Disorders and Hypersensitivity to Sounds. Our work contributes to the research on serious games, as well as to the treatment of the intended population, through their use of Sêntimus.
\end{abstract}

Resumo. Este trabalho apresenta a pesquisa envolvida no projeto e avaliação do Sêntimus: um Jogo Digital Musical para Crianças com Hipersensibilidade Sonora e Características dos Transtornos do Neurodesenvolvimento. Para sua elaboração identificamos na literatura princípios relevantes para o tratamento de ambas as patologias. As avaliações foram realizadas com 5 musicoterapeutas e com 3 crianças com Transtornos do Neurodesenvolvimento e Hipersensibilidade Sonora. Nosso trabalho traz contribuições para a pesquisa sobre jogos sérios, e também de natureza prática, uma vez que o Sêntimus pode ser apropriado para o apoio ao tratamento da população alvo.

\section{Introdução}

Os jogos digitais podem ser facilmente acessados, tanto os que contêm atributos de entretenimento ou aqueles que conversam com áreas afins como educação, psicologia, música, saúde, treinamento, entre outros [Novak 2011]. Uma das áreas de interesse desta pesquisa é a de Jogos Sérios (Serious Game), termo definido como "uma competição mental, jogado com um computador de acordo com regras específicas, que usa entretenimento, para promover treinamento governamental ou corporativo, educacional, de saúde, na política pública e em objetivos de comunicação estratégica" [Ma et al. 2011].

Nosso objetivo com esta pesquisa foi desenvolver e avaliar um Jogo Sério para crianças que apresentam queixas de Hipersensibilidade Sonora (HSS) e também características dos Transtornos do Neurodesenvolvimento (TND). Os Transtornos do Neurodesenvolvimento, de acordo com o Manual Diagnóstico e Estatístico de Transtornos Mentais 5 (DSM-5), são definidos como um grupo de condições irregulares que acontecem durante 
o desenvolvimento, antes mesmo do período escolar [Association 2014]. Estas condições manifestam-se em déficits no desenvolvimento global, comprometendo a linguagem, expressividade, motricidade e cognição. O nível de comprometimento pode variar de pessoa para pessoa, assim como a presença de comorbidade que é quando uma patologia provoca o agravamento da outra [Association 2014].

A Hipersensibilidade Sonora é caracterizada por uma desordem na sensação de intensidade dos sons do dia a dia, em que sonoridades consideradas silenciosas para boa parte da população, perturbam a população hipersensível [Hazell 2002]. É uma disfunção no processamento do som no Sistema Nervoso Central, em que a cóclea é perfeitamente normal. Sons corriqueiros como buzina, liquidificador ou fogos de artifício, entre outros, podem causar medo no hiperacústico ${ }^{1}$. Há relatos de crise profunda de depressão com isolamento e desânimo. O tratamento tem em sua base a exposição sonora gradual aos sons sinalizados com queixas [Hazell 2002, Jüris 2013].

Esta pesquisa teve caráter prático e exploratório. O nosso primeiro passo foi uma revisão bibliográfica da HSS, TND e jogos digitais musicais para esta população. Embora atualmente existam diversos jogos voltados para crianças portadoras de TND, não encontramos nenhum jogo musical específico para crianças com HSS em coocorrência com os TND. Assim, nosso objetivo foi projetar e avaliar um jogo musical que pudesse ajudar no tratamento de crianças com queixa de HSS e características dos TND, considerando o nível de comprometimento de leve a moderado para ambas patologias.

Também fizemos revisões de artigos de natureza clínica da condição da HSS e dos TND. Com base nestes trabalhos, identificamos princípios de natureza clínica relevantes para o tratamento de HSS e TND que poderiam ser explorados em jogos. Para cada um destes princípios foram definidas estratégias de como os mesmos seriam abordados no projeto e desenvolvimento do jogo Sêntimus. Além disso, para conhecer melhor o perfil do usuário, aplicamos um questionário criado especificamente para esta pesquisa, direcionado aos pais, responsáveis ou profissionais que lidam com crianças com HSS nos TND. O questionário foi divulgado a grupos de interesse e ficou disponível para preenchimento online de 27/02/2018 a 25/05/2018.

Finalmente, uma vez que o Sêntimus foi implementado, ele foi avaliado ${ }^{2}$ sob a perspectiva de dois perfis distintos: musicoterapeutas que trabalham com crianças do perfil desejado (usuários indiretos do sistema) e crianças (usuário final). Para a avaliação com os musicoterapeutas, utilizamos o Método de Inspeção Semiótica Intermediado (MISI) [Oliveira and Prates 2018]. A escolha pelo método vem da possibilidade de obter a visão do usuário indireto (stakeholder) sobre o sistema. Cinco musicoterapeutas participaram do experimento. Por sua vez, a avaliação com o usuário final envolveu a observação de uso do jogo por 3 crianças com HSS e TND com idade de 6 a 8 anos, durante uma sessão de musicoterapia ocorrida no dia 21/05/2018 no Laboratório de Musicoterapia da UFMG.

Nas próximas seções apresentamos brevemente os principais trabalhos relacionados, os princípios identificados em que o projeto do jogo se baseou, o perfil dos usuários, o jogo e sua avaliação, finalizando com um resumo das contribuições deste trabalho.

\footnotetext{
${ }^{1}$ Pessoa que sofre de HSS.

${ }^{2}$ Em conformidade com a legislação de ética em pesquisa, para avaliar o jogo submetemos o projeto ao Comitê de Ética em Pesquisa da UFMG e este foi aprovado com número 77747917.8.0000.5149.
} 


\section{Trabalhos Relacionados}

Fizemos um levantamento dos Sistemas Musicais criados especificamente para pessoas com TND. Identificamos entre eles o Pequeno Mozart, um software de composição para pessoas com Paralisia Cerebral [Barbosa 2012]; o SACA, um software de integração sensorial para crianças autistas [Garcia and Miranda 2002]; e ADACA, um software educacional para crianças no Espectro do Autismo [Caminha et al. 2016]. São relatados também na literatura, experiências com utilização de softwares musicais não específicos como Theremin, um instrumento eletrônico de captação de movimento [Bergamo 2013]. Para complementar a pesquisa inicial, fizemos outra revisão buscando por Jogos Musicais Digitais criados especificamente para crianças nos TND e pudemos citar o GoLIAh, um jogo de computador com atividades de imitação para crianças no autismo [Bono et al. 2016]; o ReacTickles MAGIC um aplicativo baseado ação e reação, feito para autistas, que usa multi-touch e sensores Kinect do console Xbox [Keay-Bright 2011]; e o Pico's Adventures, um jogo sensorial destinado a crianças autistas, que permite ao jogador ver sua imagem na tela projetada em tempo real [Malinverni et al. 2017].

\section{Princípios e Estratégias para Desenvolvimento do Sêntimus}

É delicado trabalhar com jogos e saúde, principalmente com população sensível como é a desta pesquisa. Para estabelecer os princípios que nos guiaram na construção do jogo, selecionamos pesquisas dos autores Hazell e Juris, referência em HSS, que sugerem que o tratamento seja baseado na exposição sonora [Hazell 2002, Jüris 2013]. Sobre os TND utilizamos o DSM-5 [Association 2014]. A Tabela 1 apresenta de forma resumida o ponto de natureza clínica sendo tratado e o princípio que se refere a ele (identificados na literatura), e a estratégia adotada no Sêntimus para abordá-los. Note-se que os princípios e estratégias de 1 a 5 referem-se a HSS, os demais aos TND.

\section{Levantamento do Perfil do Usuário}

Não tínhamos conhecimento sobre se, ou como as crianças com TND usavam o celular ou os aplicativos. Assim, o principal objetivo do questionário foi identificar o uso da tecnologia por estas crianças. Tivemos 35 participantes - pais, responsáveis ou profissionais relacionados às crianças com HSS e TND. Dentre os resultados, destacamos a baixa relação entre diagnóstico de HSS e a presença de queixas a certos tipos de som. Os sons que foram mais assinalados como incômodos foram liquidificador, furadeira e foguetes. Dentre os 35 participantes, 26 disseram não ter diagnóstico de HSS confirmado, mas 29 disseram perceber sons de incômodo. Todos relataram que as crianças interagem com diversos aplicativos no celular todos os dias da semana. Estes resultados mostram que um jogo para celular que trabalhe sons do cotidiano pode ser adequado para esta população.

\section{O Jogo Sêntimus}

Com base nos princípios identificados e estratégias propostas, desenvolvemos o jogo Sêntimus https:// sentimus.wixsite.com/sentimus, explorando o contexto de festa de aniversário que pode ser um ambiente desafiador à criança com HSS. O objetivo é estourar balões, tocando o dedo sobre cada balão que aparece. Cada balão estourado marca um ponto e, à medida que o jogador ganha mais pontos, a intensidade do som ambiente aumenta (Figura 1). Em algumas fases a interação com outros elementos expõem o jogador a diferentes sons, ou permite que diminuam a intensidade do mesmo. 


\begin{tabular}{|c|c|c|c|}
\hline N. & $\begin{array}{l}\text { Pontos de } \\
\text { natureza } \\
\text { clínica }\end{array}$ & Princípio & Estratégia \\
\hline 1 & $\begin{array}{l}\text { Exposição } \\
\text { Sonora Gradual }\end{array}$ & $\begin{array}{l}\text { É uma técnica que consiste na exposição } \\
\text { gradual aos sons. }\end{array}$ & $\begin{array}{l}\text { Criamos uma faixa de som ambiente, como em } \\
\text { uma festa de aniversário. Quando o jogador } \\
\text { clica no balão, a intensidade desta faixa altera. }\end{array}$ \\
\hline 2 & $\begin{array}{l}\text { Valores } \\
\text { Audiométricos }\end{array}$ & $\begin{array}{l}\text { Há uma definição de frequências e intensidades } \\
\text { para realização de testes audiométricos para } \\
\text { HSS. }\end{array}$ & $\begin{array}{l}\text { Criamos um modelo de Modelo de Ruído, } \\
\text { Modelo de Intensidades e Modelo de } \\
\text { Frequências para todos os sons do jogo. }\end{array}$ \\
\hline 3 & $\begin{array}{l}\text { Aplicação de } \\
\text { Relaxamento }\end{array}$ & $\begin{array}{l}\text { Procedimento terapêutico que consiste na } \\
\text { aplicação de técnicas de relaxamento. }\end{array}$ & $\begin{array}{l}\text { Usamos a música Happy e nos apoiamos no } \\
\text { cenário do filme Minions. }\end{array}$ \\
\hline 4 & $\begin{array}{l}\text { Ativação } \\
\text { Comportamental }\end{array}$ & $\begin{array}{l}\text { Técnica para tentar reiniciar atividades } \\
\text { abandonadas devido ao medo causado pela } \\
\text { hiperacusia. }\end{array}$ & $\begin{array}{l}\text { Criamos um ambiente de festa de aniversário } \\
\text { incentivando o hiperacústico a não evitar esses } \\
\text { ambientes. }\end{array}$ \\
\hline 5 & Psicoeducação & $\begin{array}{l}\text { Procedimento didático para ensinar que fugir do } \\
\text { som pode aumentar o medo e ansiedade. }\end{array}$ & $\begin{array}{l}\text { Criamos uma documentação textual sobre o } \\
\text { jogo. }\end{array}$ \\
\hline 6 & $\begin{array}{l}\text { Capacidades } \\
\text { Mentais } \\
\text { Genéricas } \\
\end{array}$ & Manifestação de insuficiência no raciocínio. & Desenvolvemos atividade de interação simples. \\
\hline 7 & Comunicação & $\begin{array}{l}\text { Carência no uso da linguagem, da fala e da } \\
\text { comunicação social. }\end{array}$ & $\begin{array}{l}\text { A mecânica do jogo dá-se pela experiência, } \\
\text { manipulação direta em tentativa e erro. }\end{array}$ \\
\hline 8 & Coordenação & $\begin{array}{l}\text { Desmostração de inabilidade na aquisição e na } \\
\text { execução de movimentos coordenados. }\end{array}$ & $\begin{array}{l}\text { A manipulação do jogo é direta, apertando os } \\
\text { balões e personagens que surgem nas fases. }\end{array}$ \\
\hline 9 & Aprendizagem & $\begin{array}{l}\text { Presença de déficits específicos de perceber } \\
\text { e/ou processar informações com eficiência. }\end{array}$ & $\begin{array}{l}\text { A Fase } 1 \text { foi criada com o objetivo do jogador } \\
\text { aprender a mecânica de forma gradual. }\end{array}$ \\
\hline 10 & Atenção & $\begin{array}{l}\text { Manifestação de níveis prejudiciais de } \\
\text { desatenção e/ou hiperatividade-impulsividade. }\end{array}$ & $\begin{array}{l}\text { As } 4 \text { fases foram construídas a fim de } \\
\text { proporcionar o engajamento no jogo. }\end{array}$ \\
\hline 11 & Interação Social & $\begin{array}{l}\text { Presença de déficits na comunicação social e } \\
\text { interação social. }\end{array}$ & $\begin{array}{l}\text { Criamos um perfil para cada jogador ter aceso } \\
\text { ao seu desempenho. }\end{array}$ \\
\hline & $\begin{array}{l}\text { Especificadores } \\
\text { de Gravidade }\end{array}$ & $\begin{array}{l}\text { Distinção de níveis de gravidade: Leve, } \\
\text { Moderado, Grave e Profundo. }\end{array}$ & $\begin{array}{l}\text { O jogo tem opção de configuração onde pode } \\
\text { ser customizado para cada jogador. }\end{array}$ \\
\hline
\end{tabular}

Tabela 1. Princípios e Estratégias do Jogo Sêntimus
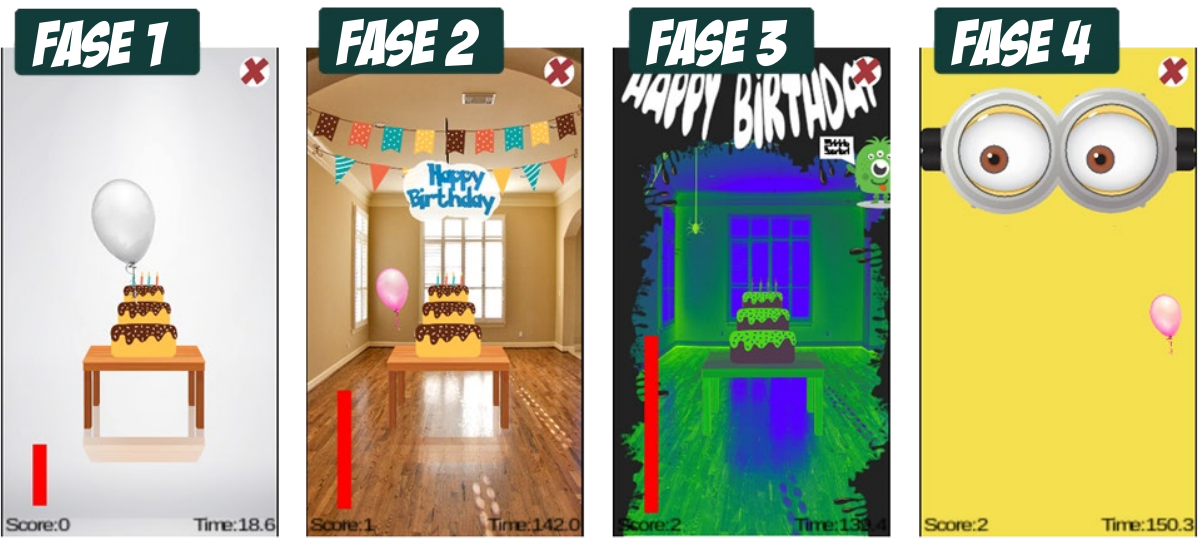

Figura 1. As 4 Fases do Sêntimus. Fase 1 Aprendendo a Mecânica, Fase 2 Festa de Aniversário, Fase 3 Fantasmas e Fase 4 Relaxamento.

\section{Avaliação com o Sêntimus}

Para avaliar o Sêntimus, realizamos dois estudos. O primeiro foi realizado com musicoterapeutas profissionais, e o segundo com crianças com HSS e TND de 6 a 8 anos.

\section{Inspeção do Sêntimus com Musicoterapeutas}

Embora os musicoterapeutas não sejam o usuário final, a quem o jogo se destina, eles são usuários indiretos importantes, uma vez que o jogo tem o objetivo de apoiar o tratamento de crianças nas condições citadas. Assim, sua análise sobre o jogo é uma 
avaliação especializada relevante sobre nossa proposta. O Método de Inspeção Semiótica Intermediado (MISI) é um método fundamentado na Engenharia Semiótica que tem por objetivo avaliar a comunicabilidade de um sistema a partir da perspectiva de um usuário indireto [Oliveira and Prates 2018]. A inspeção acontece com base em um roteiro de entrevista, guiando o participante pelo sistema. Durante a inspeção, a interação e entrevistas são gravadas (vídeo e áudio), e posteriormente o áudio é transcrito para a análise.

A partir da análise, emergiram 7 aspectos (temas) considerados relevantes pelos musicoterapeutas, dos quais destacaremos quatro: 1. A interação proposta pelo jogo era adequada, podia ser aprendida através da própria interação, tendo em vista a simplicidade; 2. Os sons do jogo foram considerados apropriados pois é o tipo de som que mais provoca evasão de uma criança com HSS no seu dia a dia; 3 . A customização do jogo foi imprescindível para uso musicoterapêutico, pela possibilidade de adaptação a cada jogador; 4. O jogo pode ser um apoio no tratamento da HSS, porém o uso deve ser feito com orientação. De acordo com as declarações das terapeutas, concluímos que o jogo é válido como ferramenta de dessensibilização e seu uso pode trazer impactos reais no cotidiano do jogador. Os resultados mostraram que os musicoterapeutas entenderam as estratégias (e.g. o uso de variação de intensidade sonora durante as fases do jogo) e as respaldaram ao discutir a sua relevância na interação da criança com o jogo, ou um potencial uso do Sêntimus como parte de atividades de musicoterapia.

\section{Avaliação do Sêntimus com Crianças}

A avaliação foi realizada com 3 crianças, duas diagnosticadas com autismo e uma com Síndrome de Down. Fizemos o recrutamento com lista de espera do Laboratório de Musicoterapia da UFMG. A avaliação foi feita em um único dia, em uma sessão de musicoterapia, de 30 minutos de duração, na qual o Sêntimus era uma das atividades. A sessão contou com a participação de uma musicoterapeuta, guiando a criança, e da autora como observadora. Ambas fizeram anotações no formulário de observação durante a sessão. Outros 2 bolsistas participaram, auxiliando a musicoterapeuta e 1 bolsista filmando. Cada participante jogou pelo menos uma vez todas as fases do jogo, e um deles jogou 3 vezes consecutivas. Fizemos uma análise descritiva sobre cada jogador a partir dos dados contidos em um formulário de observação preenchido durante o procedimento. O resultado foi favorável para indicações de expressões positivas na experiência com o jogo, porém não nos dizia muito sobre engajamento. Por esta razão partimos para a investigação dos dados de desempenho armazenados no sistema, em contraste com as 4 dimensões do método ARCS [Keller 1987]. Assim, obtivemos respaldo quanto aos indicativos de engajamento do jogador (e.g. pontuação adquirida, balões estourados, nível sonoro alcançado, entre outros), nas dimensões atenção, relevância, confiança e satisfação. Os dados indicaram que os jogadores estavam engajados e reagiram positivamente à experiência.

\section{Considerações Finais}

Este trabalho traz contribuições para diferentes áreas de pesquisa como computação, musicoterapia ou de natureza mais interdisciplinar. Tratar de HSS e TND juntas, mostrou-se uma iniciativa inédita em relação aos trabalhos relacionados desta investigação e desafiadora na organização e desenvolvimento dos requisitos do jogo. Assim, a revisão bibliográfica realizada sobre jogos sérios específicos para HSS e TND contribui para pesquisadores com interesse nesta área. Além disso, a identificação dos princípios de tratamento 
de HSS e estratégias apresentadas podem ser usados na proposta de novos recursos e jogos voltados para crianças com HSS. A metodologia aplicada no projeto e avaliação conduzidos na nossa pesquisa pode ser replicada ou adaptada para o desenvolvimento de jogos sérios voltados para a saúde de forma geral. Finalmente o Sêntimus é uma contribuição prática deste trabalho, uma vez que está implementado e pode ser utilizado de forma gratuita por pessoas interessadas. A sua avaliação com as musicoterapeutas e com as crianças indicam o potencial prático do seu uso no apoio ao tratamento de crianças hiperacústicas. Os próximos passos do nosso projeto envolvem a proposta e desenvolvimento de novas fases para o jogo, considerando outros contextos cotidianos, e também uma avaliação de seu uso a mais longo prazo por crianças hiperacústicas por meio da musicoterapia.

\section{Agradecimento}

Aos participantes do questionário, musicoterapeutas, às crianças e seus responsáveis que contribuíram para pesquisa, e também à CAPES e ao PIPA/UFMG pelo financiamento.

\section{Referências}

Association, A. P. (2014). DSM-5: Manual Diagnóstico e Estatístico de Transtornos Mentais.

Barbosa, S. M. T. (2012). Software pequeno Mozart: uma porta para a música: um estudo de caso na paralisia cerebral. B.S. thesis.

Bergamo, H. (2013). O Uso de Ferramentas Tecnologias no Contexto Da Educação Musical e da Musicoterapia. Anais do XV Fórum Paranaense de Musicoterapia.

Bono, V., Narzisi, A., Jouen, A.-L., Tilmont, et al. (2016). Goliah: a gaming platform for home-based intervention in autism-principles and design. Frontiers in Psychiatry.

Caminha, V. et al. (2016). Autismo: vivências e caminhos. São Paulo: Blucher.

Garcia, D. F. and Miranda, J. (2002). Um software de apoio à melhoria da interação de crianças com características autistas. Brazil Symposium on Computers in Education.

Hazell, J. (2002). Hypersensitivity of hearing: hyperacusis, phonophobia and recruitment. AIA Newsletter, Autumn.

Jüris, L. (2013). Hyperacusis Clinical Studies and Effect of Cognitive Behaviour Therapy. $\mathrm{PhD}$ thesis, University Hospital, Akademiska.

Keay-Bright, W. (2011). Designing interaction though sound and movement with children on the autistic spectrum. In International Conference on Arts and Technology.

Keller, J. M. (1987). Development and use of the arcs model of instructional design. Journal of instructional development.

Ma, M., Oikonomou, A., et al. (2011). Serious games and edutainment applications. Springer.

Malinverni, L. et al. (2017). An inclusive design approach for developing video games for children with autism spectrum disorder. Computers in Human Behavior.

Novak, J. (2011). Game development essentials: an introduction. Cengage Learning.

Oliveira, E. R. d. and Prates, R. O. (2018). Intermediated semiotic inspection method. In Proceedings of the 17th IHC. 